\title{
CAUCHEMARS CULINAIRES, NAUSÉE NARRATIVE LES ENJEUX ESTHÉTIQUES DE LA RÉTROSPECTION
}

\author{
Richard-Laurent Barnett*
}

Les destinées d'un peuple dépendent et de sa nourriture et son son régime (...) Moins la force humaine est occupée, plus elle tend à l'excès; la pensée l'y porte irrésistiblement (...) Il suit de là que, plus les sociétés sont civilisées et tranquilles, plus elles s'engagent dans la voie des excès.

Honoré de Balzac

Une fois, je me suis fait un soufflé au fromage: après deux cuillerées, c'était fini.

Simone de Beauvoir

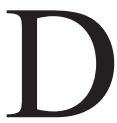

ans la lutte viscérale que se livrent depuis la nuit des temps les Gras et les Maigres, on pouvait légitimement s'attendre à ce que les Modernes se rangent du côté de la minceur. Partisans convaincus du frugal, du non-frelaté, et d'un mode de préparation qui traite les ingrédients d'un mets en exaltant leur quant à soi gustatif souligné d'ailleurs par le caractère "discret" de leur disposition sur l'assiette, ces petits mangeurs ne pouvaient que pactiser avec la Nouvelle Cuisine contre la grande tradition gastronomique française. Culte bourgeois du copieux, science inégalée des apprêts: cela déjà aurait suffi à la leur faire prendre en grippe. Mais ce qui les offusque surtout dans son style d'accommoder les nourritures, c'est la place royale qu'elle réserve au "lié" et à l'onctueux. Comme en témoigne littérairement le fameux boeuf en daube ou "boeuf mode" proustien, son plus beau titre de gloire ne réside-t-il pas dans la spécialité qu'elle s'est faite de fondre, et par là même de rendre méconnaissables,

* George Southwers Tern State University. Frederick A. Treuhaft. Senior Research Fellow. Ribarnett@msn.com 
BARNETT, R-L. Cauchemars culinares, nausée narrative...

les aliments naturels qui entrent dans la composition d'un plat - le grand art consistant précisément à atteindre cette homogénéité délicieuse qui estompe leur identité d'origine ou, plutôt, qui la "transcende" en faisant, aussi subtilement que possible, passer formes et saveurs les unes dans les autres?

Or, on serait bien en peine - le voudrait-on - de prendre exemple sur Jean-Pierre Richard ${ }^{1}$ pour tenter, à sa manière, de reconduire sur les écrivains des années 50 et 60 les analyses inoubliables qu'il a consacrées naguère à l'imaginaire nutritif d'un Flaubert, d'un Huysmans, ou d'un Proust. Non seulement faute d'une documentation suffisante (si on mange peu chez les Modernes, on parle de manger encore moins). Mais surtout parce que leur idiosyncrasie alimentaire, pour autant qu'on puisse s'en faire une idée, semble dominée par les aversions et les phobies bien davantage que par les plaisirs de table ou l'heureuse appétence.

Le cas des nouveaux romanciers et de leurs comparses porte l'empreinte indissoluble de cette réticence: quand la rêverie substantielle n'est pas d'emblée tenue en échec par la description distanciée (purement optique, objectale, géométrique et technologisée) d'un Robbe-Grillet se plaisant à réduire une rondelle d'oeuf, une tranche de pain, ou un quartier de tomate à sa version aseptisée et prophétiquement fast food,$^{2}$ la scène de repas à laquelle il nous est exceptionnellement donné d'assister est, de manière symptomatique, génératrice de malaise, ${ }^{3}$ et il en va de même des quelques mentions et comparaisons culinaires rencontrées au cours d'autres lectures. Spasmes ressentis au spectacle de la "viande en sauce" dans L'Amante anglaise, où l'appréhension panique d'un ragoût baignant dans cette "sale sauce de graisse" se trouve associée à la fois

1 Proust et le monde sensible (Paris: Seuil, 1974). Consulter en supplément: REVEL, J-F. La sensibilité gastronomique de l'Antiquité à nos jours. Paris: Surger, 1985; ARON, J-P. Le mangeur au XIXème siècle. Paris: Laffont, 1973; GUILLEMARD, C. Les mots de la cuisine et de la table. Paris: Belin, 1990.

2 On trouvera cette description célèbre (et dont le puissant effet de rupture a été relevé notamment par R. Barthes) dans son roman Les Gommes (Paris: Ed. de Minuit, 1953. p. 160 et passim). L'analyse du fonctionnement contextuel des invocations culinaires dans la littérature contemporaine, bien que le plus souvent absente des travaux critiques, trouvent pourtant sa place (comme insidieusement) dans un certain nombre d'études qui valent notre attention: ARIÈS, P. La fin des mangeurs: les métamorphoses de la table à l'âge de la modernisation alimentaire. Paris: Desclée De Brouwer, 1997; BENS, J. La cuisine en jeux. Paris: Zulma, 1998; FERET-FLEURY, C. Le gout et les mots. Paris: Gallimard, 1996; SOLLERS, P. La guerre du goût. Paris: Seuil, 2000; JOST, P. La gourmandise. Paris: Le Pré Aux Clercs, 1998; et BARTHES, R. Mythologies. Paris: Seuil, 1957.

3 Je songe au dîner décrit au chapitre VII de Moderato Cantabile de Marguerite Duras et dont l'héroïne, prise de vertige devant le saumon à la mayonnaise et la chair trop grasse du canard à l'orange, trouble la cérémonie. 
au corps difforme - si gros et obtus qu'on en manque d'air - de l'infortunée Marie-Thérèse et, par contraste avec cette densité et cette mauvaise lourdeur, à la plante éponyme du roman, à laquelle on prête des vertus sédatives; "éclairs au chocolat, babas et tartes" qui, dans le texte X de Tropismes, trahissent par assonance et métonymie le désir régressif de s'agglutiner et de disparaître dans la masse à l'heure du thé; repas interminables dans Les Choses, où "rôtis et volailles ne s'accompagnaient d'aucune sauce"; ${ }^{4}$ horreur persistante du nappé qui empoisse, des Mythologies jusqu'à La Chambre claire: qu'ils soient empruntés à Marguerite Duras, Nathalie Sarraute, Georges Perec, ou Roland Barthes, ces exemples dysphoriques (parmi lesquels il est impossible, compte tenu de l'investissement fantasmatique auquel ils donnent lieu, de ne voir que la simple contepartie négative de ce régime sec dont Nietzsche vante la tonicité dans Ecce homo), font preuve d'une remarquable convergence en matière de tabou et de dégoût: de fácon répétitive et insistante, ils attestent la même répugnance pour une nourriture mijotée, trop riche ou, plus exactement, engorgée.

Davantage: si "on peut dire, avec raison, que c'est le jus qui sépare le registre gras du maigre", 5 ils font apparaître que ce n'est pas tant le jus que la sauce qui, pour les Modernes, mue la nourriture en quelque chose d'immonde: dans la mesure où il englue d'avance son consommateur à la faveur d'une participation imaginative contre laquelle celui-ci ne saurait se défendre, il est aisé de comprendre qu'un aliment suintant de graisse ou noyé dans sa sauce ne puisse que faire suffoquer, provoquer la nausée, et apparaître à un adepte de la légèreté comme l'épiphanie culinare de l'obscène.

4 Repas si parfaitement illustratifs que le passage vaut d'être cité en son entier: "Ils mangeaient et buvaient pendant des heures entières. L'exubérance et l'abondance de ces repas étaient curieuses: à vrai dire, d'un strict point de vue culinaire, ils mangeaient de façon médiocre: rôtis et volailles ne s'accompagnaient d'aucune sauce: les légumes étaient, presque invariablement, des pommes de terre sautées ou cuites à l'eau, ou même, en fin de mois, comme plats de résistance, des pâtes ou du riz accompagné d'olives et de quelques anchois. Ils ne faisaient aucune recherche; leurs préparations les plus complexes étaient le melon au porto, la banane flambée, le concombre à la crème. Il leur fallait plusieurs années pour s'apercevoir qu'il existait une technique, sinon un art, de la cuisine, et que tout ce qu'ils avaient par-dessus tout aimé manger n'était que des produits bruts, sans apprêt ni finesse." (PEREC, G. Les Choses. Paris: Julliard, 1965. p. 57-58).

5 BRIllat-SAVARIN, J. A. Physiologie du goût. Méditation V. Différence du gras au maigre. Paris: Sautelet, 1828. t. 1, p. 152). Voir aussi: ABENSOUR, C. Nourritures: Zola, Proust, Valéry, Ponge. Paris: Quinette, 1990; GILLET, P. Le goût et les mots. Paris: Payot, 1987; ROUANET, M. Paroles de gourmandise. Paris: A. Michel, 1998; et TRÉBAOL, F. Mots en bouche. Paris: Ed. du Carrousel, 1998. 
Ce cauchemar nutritif - que pourrait d'ailleurs conforter l'imaginaire architectural des Modernes ${ }^{6}-$ ne nous aurait pas retenu ici, on s'en doute, s'il ne pouvait être considéré comme l'expression d'une esthétique et d'une poétique à peine déguisées. Comme l'écrit Maupassant, "les gras n'ont pas le droit de se servir des mêmes mots et des mêmes phrases que les maigres", ${ }^{7}$ et l'interdiction conserverait certainement toute sa validité si on l'étendait au domaine de la philosophie et à la sphère politique...

Pour nous en tenir ici à la complicité spéculaire des mets et des mots, citons à titre d'exemple un passage du Vent de Claude Simon, que notre bref échantillonnage aurait déjà pu alléguer précédemment:

Car, me dit-il, ce fut ainsi que cela se passa, en tout cas ce fut cela qu'il vécut, lui: cette incohérence, cette juxtaposition brutale, apparemment absurde, de sensations, de visages, de paroles, d'actes. Comme un récit, des phrases dont la syntaxe, l'agencement ordonné - substantif, verbe, complément - seraient absents. Comme ce que devient n'importe quel article de journal (le terne, monotone et grisâtre alignement de menus caractères à quoi se réduit, aboutit toute l'agitation du monde) lorsque le regard tombe par hasard sur une feuille déchirée qui a servi à envelopper la botte de poireaux et qu'alors, par la magie de quelques lignes tronquées, incomplètes, la vie reprend sa superbe et altière indépendance, redevient ce foisonnement désordonné, sans commencement ni fin, ni ordre, les mots éclatant d'être de nouveau séparés, libérés de la syntaxe, de cette fade ordonnance, ce ciment bouche-trou indifféremment apte à tous usages et que le rédacteur de service verse comme une sauce, une gluante béchamel pour relier, coller tant bien que mal ensemble, de façon à les rendre comestibles, les fragments éphémères et disparates de quelque chose d'aussi indigeste qu'une cartouche de dynamite

6 Sur la haine moderne de la dalle, du bloc, du crépi et d'un type de restauration qui noie dans le béton l'appareil des murs, voir DÄLLENBACH, L. Claude Simon. Paris: Ed. du Seuil, 1987. (Coll. Les Contemporains) p. 124 et passim; BOURDIEU, P. La Distinction. Paris: Ed. de Minuit, 1979; et BARNETT, R-L. Offuscation et transparence, ou le triomphe de l'obstacle. Studi di Letteratura Francese, p. 77-94, 2002.

7 MAUPASSANT, G. de. Lettre trouvée sur un noyé. In: Contes et Nouvelles. Paris: Albin Michel, 1957. t. 2, p. 904. Citation qui fait penser à certains passages de La Bruyère. 
ou une poignée de verre pilé: grâce à quoi (au grammairien, au rédacteur de service et à la philosophie rationaliste) chacun de nous peut avaler tous les matins, en même temps que les tartines de son petit déjeuner, sa lénifiante ration de meurtres, de violences et de folie ordonnés de cause à effet, quitte, si cela ne le satisfait pas (et apparemment, et contrairement à ce qu'il pense, cela ne le satisfait pas), à recourir en supplément aux bons offices des esprits, du marc de café, des cierges bénis, des hommes providentiels ou de la camisole de force.

Dans son récit donc, ou plutôt chaque fois qu'il me parla plus tard de ces journées (car ce ne fut que par bribes qu'il me raconta tout cela, et peu à peu, et non pas à proprement parler sous la forme d'un récit ... .). ${ }^{8}$

Or, s'agissant de récit, et plus précisément de récit littéraire, il ne fait aucun doute que s'il lui fallait mettre un nom sur l'écrivain qui se rapproche le plus, à son sens, de ce "rédacteur en chef" expert dans l'art de dissoudre la discontinuité du vécu dans la béchamel et de colmater les trous perceptifs et mémoriels avec du ciment prompt, le premier à venir à l'esprit d'un Moderne serait sans hésitation, celui de...Balzac! ${ }^{9}$

La comédie humaine: surchargée, bétonnée, enlisée et écoeurante à vomir? Son génial Auteur: grand Cimentier devant l'Eternel Maître Queux de cette cuisine en sauce si chère aux notaires de province immortalisés par son oeuvre que Simon lui-même, dans son roman, met en scène? ...

Pour ébahissant que cela risque de paraître aujourd'hui avec le recul dont nous disposons, telle est bien en effet l'image que le Nouveau Roman et ses compagnons de route se sont faite de Balzac et, surtout, qu'ils ont propagée

8 SIMON, C. Le Vent. Tentative de restitution d'un retable baroque. Paris: Ed. de Minuit, 1957. p. 174 et passim).

9 Il est en quelque sorte bizarre que Balzac s'avère le seul adversaire explicite, pour ainsi dire, en plein champ de bataille face aux Modernes - alors que Zola, Huysmans, Perec, Proust, Flaubert, Maupassant, Apollinaire et maints autres écrivains ne sont pas moins coupables des actes ostensiblement felonieux dont on accuse le pauvre Honoré - sans revendication aucune. C'est sans doute en fonction de l'immense facture de sa production (= ou, en termes, culinaires, l'épaisseur intense et gluante de la sauce romanesque) que l'univers textuel de Balzac adopte un statut spécial et se prête presque trop facilement aux reprises dénigrantes - sinon parfois délirantes - auxquelles son oeuvre est - il faut y succomber incessamment assujettie - sans fin, sans fond. On lira à profit l'ouvrage de COURTINE, R. Balzac à table. Paris: Laffont, 1987. 
pour se faire eux-mêmes valoir. ${ }^{10}$ A la notable exception de Butor qui l'a toujours considéré comme un tremplin pour aborder en meilleure connaissance de cause les problèmes qui se posent à la littérature d'aujourd'hui, ils n'ont eu de cesse de prétendre que la statue surdimensionnée et inusable de ce baba cool des Lettres leur bouchait l'horizon, et de l'estampiller lui-même comme "le mauvais objet" culinaire et littéraire par excellence-le parangon de cette littérature trop pleine ${ }^{11}$ dont l'obésité adipeuse et satisfaite provoque infailliblement des hauts de coeur.

Si cela revient à dire que la "bouffissure" à laquelle ils se montrent si allergiques tient à l'obsession balzacienne de la complétude et aux moyens que le romancier met en oeuvre dans son souci de ne jamais être pris en défaut, qu'est-ce, concrètement, qui révulse les Modernes, et sur quoi se focalise leur unanime exécration? ${ }^{12}$

Entre autres péchés dont Balzac (symbole abusé, lui, de tout un cortège d'illustres romanciers) se serait rendu coupable, il est trois tares que les vingtiémistes (et surtout ceux de la deuxième moitié du siècle) considèrent comme particulièrement répulsives: la facilité avec laquelle il recourt au principe de causalité pour tout expliquer et pour relier son texte en exploitant les codes d'une espèce de "colle logique"; l'intarissable blabla - autre forme de glu dont il use également sans vergogne pour tisser des liens analogiques plus que douteux entre le particulier et le général et remplir les espaces interstitiels de ses romans quand il a l'impression qu'ils bâillent ${ }^{13}$ les garanties extra-littéraires (du type "bons offices des esprits, du marc de café, des cierges bénis, des hommes providentiels", pour reprendre l'énumération à l'emporte-pièce de Claude Simon)

10 Phénomène qui figure au sein de quelques traités exégétiques: FLANDRIN, J. L. Histoire de l'alimentation. Paris: Flammarion, 1996; FENS-ARMELAGOS, J. Anthropologie des coutumes alimentaires. Paris: Denoël, 1985; CHATELET, N. Le corps à corps culinaire. Paris: Seuil, 1977; et CHATELET, N. Histoires de bouche. Paris: Folio, 1988.

11 Omniprésente dans l'étude que Roland Barthes consacre à Sarrasine (qui, comme par hasard, met en scène la castration), cette horreur atteint son paroxysme avec ce constat: "Le texte est plein de sens multiples, discontinus et entassés, et cependant poncé, lissé par le mouvement 'naturel' de ses phrases: c'est un texte-oeuf' (Cf. BARTHES, R. S/Z. Paris: Ed. du Seuil, 1970. p. 90-91), à moins qu'une série de comparaisons fantasmatiquement chargées ne fasse monter le dégoût d'un degré encore lorsque la Pleine littérature se retourne "en littérature qui est pleine: comme une armoire ménagère où les sens sont rangés, empilés, économisés (...), comme une femelle pleine des signifiés dont la critique ne se fera pas faute de 1'accoucher" (BARTHES, 1970, p. 91-92).

12 Haine (ou extrême déplaisir) qui se manifeste par moments dans les écrits de Jean Baudrillard, Roland Barthes at Pierre Bourdieu, entre autres. On ne saurait ne pas faire mention du texte magistral de LÉVY-STRAUSS, C. Le cru et le cuit. Paris: Plon, 1964; qui, de sa propre manière, encode le comble de cette négativité émotive et textuelle.

13 L'expression est de BARTHES, $S / Z$, op. cit., p. 162. 
qu'il s'octroie libéralement pour légitimer une fiction et une écriture qui, ne fonctionnant jamais sans filet, refusent obstinément de s'assumer comme telles.

Aussi éprouvent-ils sans relâche le besoin d'en découdre avec le "roman balzacien" et estiment-ils que le meilleur service que la critique puisse rendre à La Comédie humaine consiste à la désengorger en l'atomisant en lexies,${ }^{14} \mathrm{et}$ en la décapant de sa colle forte et de sa mauvaise graisse,${ }^{15}$ ou de manière plus brutale encore, en ne retenant que deux nouvelles parmi ses cent et quelques titres: Sarrasine, puisque Barthes a daigné s'y intéresser, fût-ce pour "enfoncer" Balzac plus encore; et Le Chef d'oeuvre inconnu, parce que ce texte thématise explicitement l'inéluctabilité du fragmentaire et que, par là même, il offre des gages à la Modernité... ${ }^{16}$

Qu'il faille voir dans cette réception bloquée ou, pour le moins, astringente et sélective, la conséquence directe de ce "portrait de l'artiste en Totalisateur" que le Nouveau Roman a monté en épingle et fini par populariser, c'est là une évidence que confirmerait au besoin la figure antithétique de Frenhofer et la gloire dont a joui, pendant ces années d'opprobre, le champion du fragment qu'il était devenu. Quant à savoir si, et dans quelle mesure, cette image et cette mise au pilori étaient fondées, cette question outrepasse les limites du présent contexte. Elle signale plutôt un autre itinéraire exégétique et n'entre pas (directement) en jeu ici. Non pas tant parce qu'elle m'a occupé déjà à plus d'une reprise, ${ }^{17}$ ou que je la tiendrais pour obsolète après la fin de la mise en quarantaine. Mais parce que dans l'optique adoptée, l'intérêt du rejet dont le romancier et son oeuvre ont si durablement pâti ne réside pas dans le bien-fondé ou, au contraire, dans le caractère contestable des motifs qu'on pourrait invoquer à

14 A ce bavardage impénitent dont il a été le premier à mettre en évidence le caractère narrativement intéressé, Gérard Genette oppose, comme on sait, les "silences de Flaubert", qui font de lui le "premier des écrivains modernes" (cf. GENETTE, G. Figures. Paris: Ed. du Seuil, 1966. p. 223-243; et GENETTE, G. Figures II. Paris: Ed. du Seuil, 1969. p. 78-86).

15 Comme Barthes s'y emploie dans $S / Z$.

16 Lucien Dällenbach offre, sous ce rapport, des commentaires subtils et imagés: Le tout en morceaux. Poétique, n. 42, p. 207-231, 1980; commentaires autrement repris et renchéris par BARTHES, R. L'Obvie et l'obtus. Paris: Gallimard, 1982; et dans plusieurs segments des Oeuvres complètes de Georges BATAILLE (Paris: Gallimard, 1987).

17 BARNETT, R-L. Tropismes sémiologiques. Littératures, Toulouse-Le Mirail, n. 54, p. 111-127, 1999; BARNETT, R-L. Poétique des marges: lectures d'excentration. Paris: Presses Universitaires du Nouveau Monde, 2003; et BARNETT, R-L. Narration et travestissement. Neophilologus, numéro spécial, p. 107-120, 2003; BARNETT, R-L. Textes anorectiques. Degré Second, n. 37, p. 88-116, 2001. L'ouvrage de DÄLLENBACH, L. La Canne de Balzac. Paris: Corti, 1996; offre des observations tant pénétrantes que nouvellement contournées. 
BARNETT, R-L. Cauchemars culinares, nausée narrative...

son propos, mais dans le fait qu'il signifie comme tel et tire à conséquence pour un texte littéraire dans la mesure où il permet d'en spécifier $a$ contrario l'idéal moderne.

A partir du cadastre du désirable et, surtout de l'indésirable que nous avons hâtivement dressé, on peut en effet soutenir que pour correspondre au goût de la Modernité, une oeuvre doit remplir trois conditions essentielles et doit se conformer à cette triple prescription.

La première est l'obligation d'affirmer son identité fragmentaire, et cela non pas seulement en se présentant comme une mosaïque - c'est-à-dire comme un montage discontinu de pièces détachées -, mais en soulignant de surcroît que cet agencement de fragments est lui-même lacunaire en ce sens qu'il lui manque ou une plusieurs pièces pour être complet. Un fragmentaire, donc, qui dérive justement d'une incomplétude requise, impérativement insoluble. Or, à la différence de ce qui se passait chez Balzac, cette absence s'avère désormais non substituable, puisque ce qui compte par-dessus tout aux yeux des Modernes, c'est de réaliser une mosaïque infaisable, - autrement dit: une mosaïque d'autant plus crédible, au plan esthétique, qu'elle résistera efficacement à toute tentative - si joueuse soit-elle - de totalisation et d'unification en termes de sens. Aussi est-il conforme à cette logique que ses auteurs se traguent de ne pas boucher les trous ${ }^{18}$ ou qu'ils avouent même, lorsqu'ils ont affaire à un objet trop compact, ne pas hésiter à se munir d'un marteau, d'un couteau ou d'un scalpel chirurgical.

Comme en témoigne allégoriquement dans L'Amante anglaise le corps dépecé de la trop massive Marie-Thérèse (dont le sort, par ailleurs, n'est pas sans évoquer les sévices qu'on rêve d'infliger au corpus boursouflé de $\mathrm{La}$ Comédie humaine ${ }^{19}$ ), le Tout est instinctivement perçu à la fois comme une farce et une menace par les Modernes, ${ }^{20}$ quant à l'unité, ils sont unanimes à la ressentir comme ersatz, fake, artificieux, comme un soi-disant "objet" inauthentique et suspect. On s'explique donc que le Tout Un ait chez eux pour effet de libérer la pulsion agressive et d'appeler en riposte, comme ici, le geste tranchant.

18 Motif qui, chez certains d'entre eux (notamment Claude Simon), devient une véritable rengaine.

19 Le rapport est même explicite dans le commentaire que Marguerite Duras fait de son film La Femme du Gange: un film "comblé d'images", "sans faille", "sans trous", où "tout est explicite", "bouché", "asphyxiant", "digéré", "vomitif" ou "balzacien" pour tout dire, pousse au crime... Cf. DURAS, M. Les Parleuses. Paris: Ed. de Minuit, 1974. p. 86 et passim).

20 Sentiment que Roland Barthes résume par la formule: BARTHES, R. La Totalité tout à la fois fait rire et fait peur. In: Roland Barthes par Roland Barthes. Paris: Ed. du Seuil, 1975. p. 182. A cet égard, les analyses perspicaces de Jan Baetens pèsent: BAETENS, J. L'éthique et la contrainte. Louvain: Peeters, 1995. 
Deuxième condition à satisfaire: réaliser un texte sans sauce, qui laisse respirer matériaux et matières au lieu de les étouffer en les recouvrant d'une épaisse maïzena ou en les noyant dans le béton. Comme le révèlent la pratique du dernier Cézanne, telle toile de Miró, ou dans un autre registre la cuisine japonaise, ${ }^{21}$ pourquoi s'évertuer à relier les éléments en présence par des transitions grises ou quelque "douteuse sauce gribiche"? 22 Ce remplissage des espaces intercalaires ne pourrait que nuire à la qualité des rapports que suffisent à instaurer quelques touches pigmentées, quelques taches de couleur, ou quelques fragments de nourriture. Or selon Mallarmé qui, à cet égard aussi, s'affirme comme l'un des plus sûrs et des plus lucides révélateurs du goût moderne, il doit en aller de même en littérature: pour être esthétiquement recevable, un texte ne doit pas avoir peur du vide.

Raison des intervalles, ou blancs - que le long article ordinaire de revue, ou remplissage, indique, forcément, à l'oeil qui les prélève par endroits, cependant, quelques écailles d'intérêt pourquoi ne pas restreindre à ces fragments obligatoires où miroita le sujet, puis simplement remplacer, par l'ingénuité du papier, les transitions quelconques? $?^{23}$

Or admettre la nécessité des blancs et des ruptures quitte à donner l'impression de l'inachevé comme on l'a reproché à Cézanne à l'époque, implique de choisir entre deux postures antagonistes, entre deux termes d'une triste alternative: ou opter sans compromission pour un texte dé-lié qui, comme dirait Freud, consacre le triomphe des "processus primaires"; ou s'appliquer à faire lien sans liant, c'est-à-dire en identifiant d'autres agents de connexion que la chronologie, la causalité, la psychologie, ou le flux du discours. C'est là, comme on sait, la voie dans laquelle se sont engagés de plus en plus résolument certains

21 Cf. BARTHES, R. L'Empire des signes. Genève: Skira, 1970. p. 21-39. Si la cusine japonaise exerce une telle séduction sur Barthes, c'est "d'abord parce que sur la table, sur le plateau, la nourriture n'est jamais qu'une collection de fragments, dont aucun n'apparaît privilégié par un ordre d'ingestion: manger n'est pas respecter un menu (un itinéraire des plats), mais prélever, d'une touche légère de la baguette, tantôt une couleur, tantôt une autre... (p. 33 et passim).

22 BARTHES, Roland Barthes par..., op. cit., p. 180.

23 MALlARMÉ, S. Oeuvres completes. Paris: Gallimard (Pléiade), 1945. p. 1576. 
nouveaux romanciers pour qui seuls garantissent en définitive la valeur littéraire d'un texte les rapports qualitatifs sur lesquels repose sa composition et qui procèdent, pour l'essentiel, de la parenté sensorielle ou de la polysémie de ses constituants.

Troisième prescription, enfin, à laquelle les morceaux éparpillés du cadavre de Marie-Thérèse peuvent à nouveau servir d'emblème, et d'autant plus éloquemment qu'il est amputé de son chef introuvable: le texte-mosaïque moderne est idéalement une femme cent têtes en ce sens qu'il ne comporte pas de pièce capitale et vise à promouvoir une stricte équivalence hiérarchique entre ses unités.

Mis en lumière par un passage d'Elie Faure cité dans La Bataille de Pharsale $^{24}$ et repris positivement à son compte par Claude Simon qui en fait l'une des clauses principales de sa poétique romanesque, cet idéal démocratique, commun à la Modernité dans son ensemble, comporte une forte charge émancipatrice: abolir tout privilège entre les tesselles, c'est du même coup les inviter chacune à prendre son sort en main et donc à conquérir une marge d'indépendance toujours plus importante - d'où le caractère de plus en plus distendu et tendu de leur coexistence ainsi que du rapport - maintenant ressenti comme un joug - qu'elles entretiennent avec l'oeuvre considérée comme ensemble.

Dans ces conditions on s'explique que tel mosaïste moderne (Claude Simon par exemple, sur les traces d'Apollinaire écrivant Ondes, ou de Picasso inventant le cubisme) se passionne pour l'art africain, et que tel autre (Roland Barthes) ait redécouvert Arcimboldo après les surréalistes. Chez ce dernier, les éléments de ses paysages et de ses figures composites tendent à s'autonomiser à la manière des rébus, alors que dans la plastique nègre - si l'on en croit Jean Einstein qui, pour cette raison, y discerne, anticipés, les problèmes avec lesquels se débattent écrivains et artistes du début du $\mathrm{XX}^{\mathrm{e}}$ siècle ${ }^{25}$-, l'espace de jeu est le plus large possible et la tension, maximale, entre une statuette ou un masque

24 Evoquant les Primitifs allemands qui composent par association de motifs et mettent tout sur le même plan, Elie Faure commente: "Jamais ils ne vont par le plus droit chemin au seul essentiel et au plus logique. Le détail masque toujours l'ensemble, leur univers n'est pas continu, mais fait de fragments juxtaposés. On les voit, dans leurs tableaux, donner la même importance à une hallebarde qu'à un visage humain, à une pierre inerte qu'à un corps en mouvement, dessiner un paysage comme une carte de géographie, apporter dans la décoration d'un édifice, autant de soins à une horloge à marionettes qu'à la statue de l'Espérance ou de la Foi, traiter cette statue avec les mêmes procédés que cette horloge..." (Cf. FAURE, E. La Bataille de Pharsale. Paris: Editions de Minuit, 1969. p. 238).

25 Et bien sûr au delà, en prenant soin d'ajouter - témoin Momente de Stockhausen - que cela n'est pas vrai seulement des arts plastiques et de la littérature. 
BARNETT, R-L. Cauchemars culinares, nausée narrative...

et leurs parties constitutives. Aussi peut-on, en ce sens, appliquer au textemosaïque les termes mêmes dont se sert Friedrich Schlegel pour caractériser programmatiquement le roman romantique:

La poésie est un discours républicain; un discours qui est à luimême sa propre loi et sa propre fin, et dont toutes les parties sont des citoyens libres ayant le droit de se prononcer pour s'accorder. ${ }^{26}$

Chimère moderne - et reconnue pour telle par la Postmodernité et la Modernité finissante? Utopie mise en oeuvre, et parfois passée dans les faits? Dans la mesure où la mondialisation semble s'être donné elle aussi cette mosaïque démocratique comme ambition et image de marque, ${ }^{27}$ on reconnaîtra que la question est d'importance, et que pour se l'être posée les premiers, l'art et la littérature modernes peuvent, cette fois encore, nous servir d'irremplaçable instrument d'optique pour apercevoir les éléments d'une lutte perpétuelle, celle qui nous exhorte à confronter, comme infiniment, cet émiettement chaotique du sens. ${ }^{28}$

26 Cité par LACOUE-LABARTHE, Ph.; NANCY, J.-L. L'Absolu littéraire. Théorie de la littérature du romantisme allemand. Paris: Ed. du Seuil, 1978. p. 88.

27 Sur les modalités et les ressorts de cette reprise, voir ROGER, J. La traversée des formes. Magazine Littéraire, n. 364, p. 48-50, 1998; et STAROBINSKI, J. Le Monde physionomique. Magazine Littéraire, n. 364, p. 52-54, 1998. Pour des observations pertinentes, quoique plus abstraites, on lira à profit JENNY, L. La phrase et l'expérience du temps. Poétique, n. 79, p. 181-213, 1983.

Etude copieusement subventionnée par la Fondation Treuhaft et par le Centre National de la Recherche Scientifique (Paris) - auxquels j'adresse ma plus vive reconnaissance. A ce témoignage il faudrait certes en annexer un autre: or cet essai de synthèse, eût-il été réalísé sans le long entretien méditatif dont Laurent Jenny me fit l'amitié en mars 2003, à Genève. Trop rare privilège. 
BARNETT, R-L. Cauchemars culinares, nausée narrative...

\begin{abstract}
The essay tackles a tripartite phenomenon: 1) the ways in which "la gastronomie", as a generative cipher of societal norms and cultural prerogatives, has evolved from the $19^{\text {th }}$ to the $20^{\text {th }}$ century; 2 ) the residual effects of this evolution or "difference", which, in the sphere of textuality, largely over-determines poetic and narrative structures in literary works; and 3) the impact and consequences upon writers of the post-modern era as they come to grips with the paradoxes of contemporaneity and as they struggle to negotiate earlier texts.

Mots-clés: Post-modernité, textualité et alimentation, Balzac, le Nouveau Roman, mots et mets, les langages culinaires, littérature et gastronomie.
\end{abstract}

\title{
RÉFÉRENCES
}

ABENSOUR, C. Nourritures: Zola, Proust, Valéry, Ponge. Paris: Quinette, 1990.

APOLLINAIRE, G. Alcools. Paris: Gallimard, 1920.

ARIÈS, P. La fin des mangeurs: les métamorphoses de la table à l'âge de la modernisation alimentaire. Paris: Desclée de Brouwer, 1997.

ARON, J-P. Le mangeur au XIXe siècle. Paris: Laffont, 1973.

BAETENS, J. L'éthique de la contrainte. Louvain: Peeters, 1995.

BALZAC, H. de. La Comédie humaine. Paris: Gallimard (Pléiade), 1977.

. Traité des excitants modernes. Paris: Charpentier, 1938.

BARNETT, R-L. Narration et travestissement. Neophilologus, numéro spécial, p. 107$120,2003$.

Offuscation et transparence, ou le triomphe de l'obstacle. Studi di Letteratura Francese, p. 77-94, 2002.

Poétique des marges: lectures d'excentration. Paris: Presses Universitaires du Nouveau Monde, 2003-2004.

Textes anorectiques. Degré Second, n. 37, p. 88-116, 2001. 

1999.

BARTHES, R. La Chambre claire. Paris: Seuil, 1980.

L'Empire des signes. Genève: Skira, 1970.

. L'Obvie et l'obtus. Paris: Seuil, 1982.

. Mythologies. Paris: Seuil, 1957.

. Roland Barthes par Roland Barthes. Paris: Seuil, 1975.

S/Z. Paris: Seuil, 1970.

BATAILLE, G. Oeuvres complètes. Paris: Gallimard, 1987.

BEAUVOIR, S. de. Une mort très douce. Paris: Folio, 2000.

BENS, J. La cuisine en jeux. Paris: Zulma, 1998.

BOURDIEU, P. La Distinction. Paris: Editions de Minuit, 1979.

BRILLAT-SAVARIN, J. A. Physiologie du goût. Paris, 1828.

BUTOR, M. Improvisations sur Balzac. Paris: Editions de la Différence, 1998.

CHATELET, N. Histoires de bouche. Paris: Folio, 1988.

. Le corps à corps culinaire. Paris: Seuil, 1977.

COURTINE, R. Balzac à table. Paris: Laffont, 1973.

DÄLLENBACH, L. Claude Simon. Paris: Seuil, 1987.

La Canne de Balzac. Paris: Corti, 1996.

. Le tout en morceaux. Poétique, n. 42, p. 207-231, 1980.

DURAS, M. L'Amante anglaise. Paris: Gallimard, 1967.

Les Parleuses. Paris: Editions de Minuit, 1974.

. Moderato Cantabile. Paris: Edtions de Minuit, 1958.

FAURE, E. La Bataille de Pharsale. Paris: Editions de Minuit, 1969.

FENS-ARMELAGOS, J. Anthropologie des coutumes alimentaires. Paris: Denoël, 1985. 
BARNETT, R-L. Cauchemars culinares, nausée narrative...

FERET-FLEURY, C. Le goût et les mots. Paris: Gallimard, 1996.

FLANDRIN, J. L. Histoire de l'alimentation. Paris: Flammarion, 1996.

GENETTE, G. Figures. Paris: Seuil, 1966.

. Figures II. Paris: Seuil, 1969.

GILLET, P. Le goût et les mots. Paris: Payot, 1987.

GUILLEMARD, C. Les mots de la cuisine et de la table. Paris: Belin, 1990.

JENNY, L. La Phrase et l'expérience du temps. Poétique, n. 79, p. 181-213, 1983.

JOST, P. La gourmandise. Paris: Le pré aux clercs, 1998.

LACOUE-LABARTHE, Ph.; NANCY, J-L. L'Absolu littéraire. Théorie de la litttérature du romantisme allemand. Paris: Seuil, 1978.

LÉVY-STRAUSS, C. Le cru et le cuit. Paris: Plon, 1964.

MALLARMÉ, S. Oeuvres complètes. Paris: Gallimard (Pléiade), 1945.

MAUPASSANT, G. de. Contes et Nouvelles. Paris: Albin Michel, 1957. t. 2.

PEREC, G. Les Choses. Paris: Julliard, 1965.

PONGE, F. Le parti pris des choses. Paris: Gallimard, 1942.

REVEL, J-F. La sensibilité gastronomique de l'Antiquité a nos jours. Paris: Surger, 1985.

RICHARD, J-P. Proust et le monde sensible. Paris: Seuil, 1974.

RIFFATERRE, M. Production du texte. Paris: Seuil, 1979.

ROBBE-GRILlet, A. Les Gommes. Paris: Editions de Minuit, 1953.

ROGER, J. La traversée des formes. Magazine Littéraire, n. 364, p. 48-50, 1998.

ROUANET, M. Paroles de gourmandise. Paris: Albin Michel, 1998.

SARRAUTE, N. Tropismes. Paris: Editions de Minuit, 1957.

SCHLEGEL, F. von. Kritische und Theoretische Schriften. Berlin: P. Reclam, 1978.

SIMON, C. Le Vent. Paris: Editions de Minuit, 1957.

SOLLERS, P. La guerre du goût. Paris: Seuil, 2000. 
BARNETT, R-L. Cauchemars culinares, nausée narrative...

STAROBINSKI, J. Le Monde physionomique. Magazine Littéraire, n. 364, p. 52-54, 1998.

TRÉBAOL, F. Mots de bouche: la gastronomie. Paris: Editions du Carrousel, 1998. 